\title{
¿Se puede mejorar el aprendizaje espacial con las PAU? Un estudio de caso: la PAU de Geografía de Valencia de 2012
}

\section{Is it possible to improve spatial learning with the PAU? A case study: the PAU of Geography,}

Valencia 2012

\section{Xosé Manuel Souto Vicent Miquel Vercher Milagros Rodríguez}

Universitat de València

Resumen: Las Pruebas de Acceso a la Universidad (PAU) han sido establecidas en el sistema escolar para seleccionar el alumnado en el momento de ingresar en los estudios universitarios, pero al mismo tiempo para valorar el aprendizaje realizado en los estudios previos.

En este artículo queremos mostrar algunas evidencias de que las PAU, al menos en el caso de la Geografía, pueden servir para mejorar el aprendizaje del alumnado, además de ofrecer explicaciones pragmáticas que ayudarían a mejorar la selección del alumnado en relación con sus competencias. Todo ello gracias al estudio de una muestra representativa de exámenes que desvela el nivel cognitivo de las respuestas de los alumnos, extrayendo conclusiones muy interesantes.

Palabras clave: Evaluación, Didáctica de Geografía, programa de contenidos, aprendizaje.

Abstract: Tests of Access to University (PAU) have been established in the school system to select the student body at the time of beginning their university studies, but at the same time to assess the learning done in previous studies.

In this article, we show some evidence that the PAU, at least in the case of Geography, can serve to improve student learning and offer pragmatic explanations which would help to improve the selection of students in relation to their skills. All thanks to the study of a representative sample of tests whose result reveals the cognitive level of the student responses, drawing interesting conclusions.

Key Words: Geography teaching, assessment, syllabus, learning.

(Fecha de recepción: mayo, 2014, y de aceptación: septiembre, 2014)

DOI: 10.7203/DCES.28.3743 


\section{Introducción}

Las pruebas externas de evaluación del sistema educativo y, sobre todo, de calificación y segregación del alumnado en la progresión de sus estudios no es algo reciente en España. Las reválidas han sido un instrumento de selección del alumnado en el siglo veinte. Igualmente las Pruebas Diagnóstico y las denominadas PISA (Program for International Student Assessment) han servido para generar debates sobre la calidad del sistema educativo. En este contexto aparecen las Pruebas de Acceso a la Universidad, que no son exclusivas de España, sino que en la mayoría de los países realizan este tipo de exámenes para ordenar al alumnado respecto a sus estudios universitarios (Souto y Claudino, 2009; Klausberger, 2013, Melo, 2013).

Las pruebas externas facilitan la clasificación del alumnado, pero también permiten valorar el aprendizaje realizado en el sistema escolar previo a la Universidad y las propuestas de enseñanza que se realizan. Este doble sentido de las PAU está reconocido en la legislación española derivada de las diferentes leyes orgánicas de educación ${ }^{1}$, tal como podemos mostrar en algunos ejemplos representativos ${ }^{2}$, pues explicaban que dichas pruebas debían ser coherentes con los objetivos, contenidos y criterios de evaluación del bachillerato.

En consecuencia, la finalidad de esta prueba es doble (valorar y seleccionar) y sin esta precisión no es posible avanzar en alternativas didácticas que permitan mejorar la educación geográfica para la ciudadanía ${ }^{3}$. Sin embargo mantenemos una hipótesis básica: las pruebas de acceso están pensadas para segregar socialmente y no tanto para mejorar la calidad del sistema escolar. Por eso en este artículo queremos ofrecer una alternativa en relación con la segunda función de las pruebas: mejorar el aprendizaje geográfico.

Nuestro objetivo consiste en analizar cuál es la lógica que subyace a las PAU de Geografía en los 18 casos diferentes que existen en España ${ }^{4}$, para de este modo valorar los obstáculos que pueden suponer a la mejora del aprendizaje geográfico de los problemas sociales y ambientales, lo que es consustancial a su finalidad educativa. Pero además queremos centrarnos en el estudio de algunos tribunales representativos de la Universitat de València para estu-

\footnotetext{
${ }^{1}$ Nos referimos a las normativas que desarrollan la LOGSE (Ley Orgánica General del Sistema Educativo); LOCE (Ley Orgánica de la Calidad Educativa) y LOE (Ley Orgánica de Educación).

${ }^{2}$ Como se expresa con claridad en el Real Decreto 1025/2002, de 4 de octubre, por el que se modifica el Real Decreto 1640/1999, de 22 de octubre, modificado y completado por el Real Decreto 990/2000, de 2 de junio, por el que se regula la prueba de acceso a estudios universitarios.

${ }^{3}$ Una exhaustiva información legislativa sobre estas pruebas la podemos consultar en Martín y Vázquez (2011).

${ }^{4}$ Las 17 Comunidades Autónomas y el territorio MEC: extranjero, Ceuta y Melilla.
} 
diar las dificultades prácticas que tiene el alumnado para alcanzar una calificación que le permita elegir su futuro universitario.

Entendemos que el interés de la presenta investigación es relevante para el gremio de geógrafos y para los docentes de Secundaria. En el primer caso porque esperamos aportar orientaciones teóricas y evidencias empíricas de la manera de formular unas tareas de aprendizaje desde los protocolos de los exámenes estudiados. En el segundo caso porque pretendemos mostrar los límites y posibilidades de aprendizaje del alumnado con el conocimiento de las respuestas de casi un centenar de exámenes analizados y los informes de algunos correctores.

\section{El estado de la cuestión: las PAU y las pruebas externas}

Con este trabajo continuamos una línea de investigación personal y colectiva. En un caso se continua con un trabajo iniciado ya en los albores de este milenio, donde comparamos los obstáculos de aprendizaje en las pruebas finales de secundaria en España y Portugal (Souto y Claudino, 2001). Más tarde hemos realizado un estudio de las pruebas PAU en España y su influencia en la evaluación del sistema escolar en España (Souto, 2011; Souto 2013; Souto, Fuster y Sáiz, 2014).
Además otras personas e instituciones se han ocupado de este mismo asunto. Destacamos para nuestros intereses los trabajos de E. Climent (2001), C. Mesejo (2007), Martín y Vázquez (2011) y Navarro y Gil (2011) sobre las características de las diferentes PAU de Geografía en España, así como las de F. Quiñonero (2012) y Martínez y Gil (2011) sobre algunas circunstancias particulares de las PAU. Desde algunos departamentos universitarios, como ha sido el caso de Geografía de la Universitat de València, se han editado materiales para comentar las pruebas y los criterios de corrección y confección desde los profesores que asisten como examinadores (Obiol et al., 2008). Aunque sin duda el artículo seminal ha sido el de la profesora finesa L. Houtsonen (1988), que nos permitió trazar una metodología sobre las influencias de este tipo de pruebas en el aprendizaje posterior del alumnado.

La regulación de las PAU indica que el marco de referencia de los ejercicios será el Real Decreto que regula el curso de Geografía de segundo curso de bachillerato ${ }^{5}$. En dicho Real Decreto se indica que:

La Geografía estudia la organización del espacio terrestre, entendido este como el conjunto de relaciones entre el territorio y la sociedad que actúa en el. El espacio es para la Geografía una realidad relativa, dinámica y heterogénea que resulta de los pro-

${ }^{5}$ BOE núm. 266, Martes 6 noviembre 2007, en la página 45463 y sucesivas. 
cesos protagonizados por los grupos humanos condicionados, a su vez, por el propio espacio preexistente.

La selección de contenidos responde a este estudio de la realidad espacial de España, de sus características comunes y de su diversidad, su medio natural, los elementos que explican la diferenciación de paisajes, la plasmación de las actividades humanas en el espacio o los sistemas de organización territorial resultantes, atendiendo también a la dimensión europea de España y a su posición en el sistema mundo. Además incorpora, en un bloque inicial que debe entenderse común al resto, aquellos procedimientos característicos del análisis geográfico y técnicas que facilitan el tratamiento de datos e informaciones, así como referencias a valores que forman al alumnado en la solidaridad, el respeto y la disposición para participar activamente en su entorno espacial y social.

Sin duda, esta introducción ya nos genera una serie de preguntas, que implican sus consiguientes respuestas y toma de opciones.

Por ejemplo:

a) ¿Cómo se articulan las relaciones entre espacio, territorio y sociedad? ¿Qué diferencias conceptuales existen entre espacio y sociedad?

b) ¿Qué significa que el espacio es una realidad relativa, dinámica y heterogénea? ¿Cómo influye esta definición en la finalidad básica de la materia: aprehender y entender el espacio? ¿Aprender y aprehender significan lo mismo?

Estas cuestiones nos conducen a una hipótesis central: las PAU se realizan desde las rutinas académicas y no desde presupuestos teóricos de innovación. Además esta conjetura nos señala dos secundarias: a) la falta de investigación educativa por parte de la geografía universitaria es responsable del predominio de rutinas; b) la imagen de la geografía en la opinión pública se consolida en un cuerpo de conocimientos poco útil para resolver problemas sociales.

Sin embargo el esquema predominante de las PAU de Geografía en España muestra una manera de presentar los ejercicios que difiere mucho de los objetivos perseguidos en las finalidades declaradas en las órdenes ministeriales y autonómicas de la regulación de la materia de Geografía.

Así los criterios de evaluación marcados para la asignatura en segundo de bachillerato no aparecen en las pruebas. Los ejercicios que hemos analizado de diversas Comunidades Autónomas nos indican que no se solicita, como regla general, que el alumno interprete los documentos y elabore gráficos sobre cuestiones geográficas ${ }^{6}$, como tampoco se suele solicitar del alumnado que valore las consecuencias sobre la vida social y ambiental del proceso de urbanización.

\footnotetext{
${ }^{6}$ Algo que se indica explícitamente en los criterios de evaluación del Real Decreto mencionado en la nota anterior.
} 
Así la mayoría de los ejercicios de las PAU tienen esta estructura ${ }^{7}$ :

* Definición de conceptos.

* Localización geográfica sobre mapas mudos.

* Comentarios de gráficos, imágenes o mapas (generalmente con preguntas guía)

Vamos a utilizar las orientaciones sobre la corrección de la prueba de las universidades de Castilla-León (Prueba del año 2008), pues resume muy bien lo que se pretende:

\section{Criterios generales de correc-} ción de la prueba:

I. Tema. Se valorará, sobre todo, la coherencia en la estructura y el que los contenidos no omitan aspectos fundamentales de la cuestión.

II. Práctica. Lo correcto es leer e interpretar el material aportado y superar la mera descripción. Evite desarrollar un "tema paralelo".

III. Definiciones. Se valorará, ante todo, la exactitud, claridad y concisión de las respuestas.

IV. Localizaciones. Se exige que sean claras y precisas. No debe haber duda acerca de dónde se quiere situar el enunciado propuesto.

Como prueba empírica podemos citar estos ejemplos: Desarrollo de un tema: "Los movimientos migratorios en España y sus repercusiones territoriales". (Pregunta 3 PAU de Andalucía 2010); Vocabulario: "Defina brevemente cinco de ocho términos". (PAU Baleares 2010); Análisis geográfico (Práctica): "Comente los siguientes diagramas termopluviométricos" (PAU Cantabria 2010). Localizaciones: A la vista del mapa adjunto, "Provincias recorridas por las vías pecuarias de La Plata y Soriana Oriental", señale dichas provincias (marcadas por los dos "transectos") y la importancia histórica y actual de las mismas (Madrid, junio de 2008)

Posteriormente la AGE se reúne para establecer nuevos criterios, en especial buscando que las preguntas y los temas no se conviertan en cuestiones aisladas, obstaculizando de este modo la capacidad que posee la geografía para relacionar elementos en una explicación. Un análisis que abarca también aspectos formales sobre localización y ortografía. Es decir, existe un cierto descontento entre los profesionales de la Geografía en relación con este tipo de pruebas, lo que en el caso de Valencia ha dado lugar a algunos cursos de formación y una publicación específica ${ }^{8}$. Además cuando analizamos la prueba valenciana pode-

\footnotetext{
${ }^{7}$ Nos ha sido de utilidad el trabajo presentado por Emilia Rodríguez, María José Vázquez y Montserrat Villarino en el Seminario de Geografía de la AGE del año 2011, donde analizan todas las pruebas PAU de Geografía de los años anteriores. En este trabajo inédito (por lo que agradecemos el permiso para ser citado por parte de las autoras) se confirma la estructura aquí presentada para un total de 11 Comunidades Autónomas.

${ }^{8}$ Nos referimos a los cursos de formación para profesores de secundaria y a la publicación coordinada por los profesores Obiol, Salom y Souto (2008).
} 
mos observar algunas diferencias con el modelo dominante, a la vez que sugieren una vía alternativa.

\section{El modelo de PAU de geografía en la C.V.}

Las PAU en España se elaboran desde las comisiones organizadoras de cada comunidad autónoma, cuyas funciones son definir los criterios para la elaboración de las propuestas de examen, establecer los criterios generales de evaluación de las pruebas y coordinarse con los centros en los que se imparte el bachillerato ${ }^{9}$.

Las PAU valencianas se presentan en dos exámenes distintos, el modelo A o Ejercicio 1, y el modelo B o Ejercicio 2 , permitiendo que el alumnado pueda elegir uno u otra opción, que suelen coincidir con ámbitos temáticos de geografía física en un caso y de humana en otro.

Las pruebas valencianas presentan una estructura de examen distinta a las del resto de España porque va destinada a resolver un problema geográfico, lo que significa que las preguntas empiezan presentando el problema a través de material de apoyo como gráficos o mapas, comenzando por preguntas que irán exigiendo desde relacionar concep- tos y explicaciones a partir del material de apoyo, hasta exigir una explicación científico-explicativa del problema geográfico. Las puntuaciones por pregunta todas valen 2.5 puntos. Hemos analizado parcialmente los dos modelos del año $2012^{10}$.

El análisis de las preguntas del ejercicio 1 nos permitirá entender la secuencia de tareas en relación directa con la dificultad cognoscitiva, tal como hemos señalado anteriormente.

La primera pregunta es una pregunta sencilla, enfocada a que el alumno recuerde e identifique elementos básicos de geografía como lo son las principales unidades de relieve, sin embargo, se le incrementa la dificultad al pedirle identificar los tres tipos de roquedo, para que el alumno se fije en la relación que existe puesto que esta cuestión se encamina a mostrar una pista para el desarrollo del problema geográfico posterior del examen.

En la segunda cuestión los alumnos deberán identificar y describir las variedades climáticas que aparecen en un documento anexo, que no es un climograma, sino datos termopluviométricos. Ello es así para que se pueda redactar un texto descriptivo-explicativo coherente sin más. Mientras la anterior pregunta se encaminaba a que el alumno

\footnotetext{
${ }^{9}$ En la C.V. esta comisión la encabezan cuatro personas: un profesor de la Universidad de Alicante, otro de la Universidad de Castellón, uno de la Universidad de Valencia y otro de la Universidad Politécnica de Valencia, en representación de las cuatro instituciones.

${ }^{10}$ Los dos modelos de exámenes de la PAU de Geografía se pueden consultar en la página web de la Universitat Jaume I de Castelló (http://www.uji.es/bin/infopre/trans/examens/1112/geo.pdf) [último acceso 12 de marzo de 2014]
} 
recordara o identificara, esta vez además deberá razonar relacionando los datos con sus propios conocimientos de la materia.

En la tercera pregunta se trata de realizar un texto puramente explicativo con el mayor rigor científico posible por parte del alumno, que necesariamente deberá relacionar los documentos anexos con sus propios conocimientos sobre la materia, siendo capaz de elaborar un texto coherente, a poder ser integrando una hipótesis explicativa para darle sentido a su explicación. Es decir, se busca que el alumnado construya una argumentación integrando variables en una explicación geográfica. Por tanto hablamos de una pregunta con un salto de exigencia, que debe ser contestada con mayor nivel cognitivo frente a las demás. Está enfocada a que el alumno realice una reflexión, que será un aporte indirecto más para resolver el problema final.

La última pregunta plantea el problema geográfico final: Explicar la interacción de todas las características físicas de las cuencas de drenaje, así como la interacción entre el espacio natural, el clima y el factor antrópico. Busca, por ello, la interrelación de variables en una síntesis explicativa. Al tratarse de una pregunta abierta, el alumnado debe entender que tiene libertad en hacer todo tipo de relaciones que estime oportunas y se consideren explicaciones importantes para dar sentido al total de la explicación.

\section{Respecto al Ejercicio 2, o mode-} lo $\mathbf{B}$, vamos a comentar las preguntas de una manera más resumida, para no repetir argumentos ya expuestos. Así vemos que la primera pregunta del modelo B supone que el alumnado es capaz de comprender la información que se le presenta para poder elaborar un gráfico con los datos allí indicados. En la pregunta 2 se plantea la aplicación del conocimiento teórico en la gráfica elaborada, de tal manera que relaciona conocimientos elaborados en el ejercicio y aprendidos en clase. En la tercera pregunta deberá señalar las características del transporte ferroviario en España, elaborando una síntesis explicativa, en este caso, sobre un apartado correspondiente del temario, sin más material de apoyo que el elaborado por él o el proporcionado en el examen, habrá realizado un paso más y por tanto demostrado un aprendizaje más significativo respecto a las preguntas anteriores.

Por último en la cuarta pregunta el alumno se enfrenta al verdadero problema geográfico que el examen preparaba para él, un problema durante el cual ha ido resolviendo por partes, durante las preguntas anteriores hasta enfrentarse esta vez a su explicación final, donde deberá resolverlo comprendiendo y explicando cómo se integran y se desarrollan las redes terrestres en el espacio físico de España.

Por tanto el alumno deberá demostrar toda su valía, porque la pregunta está planteada para contestarse de forma abierta para que el alumno tenga plena libertad en relacionar todo su conocimiento con el problema geográfico. 
Como hemos podido comprobar las preguntas siguen un criterio de dificultad ascendente, pues se busca valorar la capacidad del alumnado para elaborar un conocimiento escolar; es decir, cómo utiliza los conocimientos disciplinares (en este caso Geografía) para ofrecer un discurso argumentativo con datos empíricos.

\section{La metodología de nuestro trabajo}

La metodología que hemos utilizado combina los métodos cuantitativos y cualitativos, pues la finalidad de este trabajo es mostrar empíricamente que es posible mejorar el diseño de las PAU de Geografía, para de esta manera mejorar el aprendizaje del alumnado. Como se ha indicado hemos continuado una labor iniciada hace años, donde habíamos estudiado algunos exámenes del alumnado y los protocolos de los mismos.

En esta ocasión hemos querido triangular las experiencias de los correctores de las pruebas con el análisis post facto de las respuestas del alumnado. El tercer vértice de nuestra investigación lo representan las indicaciones oficiales de la comisión de las PAU de Geografía en el País Valencià.

Por este motivo un primer análisis estadístico que hemos realizado ha consistido en escoger una muestra de exámenes representativos al total de todos los de la provincia de Valencia. Para ello, hemos obtenido del servicio de informática de la Universidad de Valencia, las notas de los exámenes de Junio de 2012 de la provincia de Valencia (desde el tribunal 12 al 26) ${ }^{11}$. Calculamos la nota media total y el intervalo de frecuencias de cada tribunal y finalmente nos quedamos con el tribunal más representativo; en nuestro caso es el tribunal 13.

A continuación, el siguiente paso suponía establecer un método de análisis cualitativo de las respuestas del alumnado, para establecer las categorías pertinentes y poder extraer conclusiones. Como se trataba de evaluar la capacidad del alumnado para resolver problemas geográficos hemos utilizado una clasificación de niveles cognitivos: las taxonomías de SOLO y Bloom.

Ambas taxonomías constituyen nuestra herramienta de análisis, puesto que son dos sistemas cognoscitivos de clasificación jerárquica que tienen hasta cinco niveles de cognición aplicables mediante unos criterios específicos, lo que permiten ser adaptados en diversos estudios sobre didáctica en educación de ciencias sociales. Sin embargo, como ahora mostraremos, las taxonomías no delimitan materialmente las cuestiones conceptuales de los protocolos y el análisis de las respuestas del alumnado, sino que se consideran una guía para entender el creciente proceso de gradación de la dificultad cognitiva deseable.

\footnotetext{
${ }^{11}$ Queremos agradecer a María José Lorente, responsable de la Universidad de Valencia para la incorporación del alumnado a la Universidad, su ayuda prestada en la realización de este trabajo.
} 
La taxonomía de Bloom ha sido utilizada para clasificar jerárquicamente el nivel cognitivo al que están enfocadas las preguntas del examen. Es decir, analizamos los protocolos de las preguntas que se formulan por la comisión de los exámenes, para comprobar la coherencia respecto a la gradación de dificultades conforme progresan las preguntas. La revisión realizada por Anderson y Krathwolhl (2001) nos ha sido de gran utilidad pues nos ha permitido seleccionar las siguientes operaciones mentales. El cuadro I nos permite sintetizar los niveles jerárquicos de esta clasificación:

\section{Cuadro I. Niveles taxonómicos de Bloom}

\begin{tabular}{|l|l|}
\hline Nivel jerárquico & Descripción de tareas cognitivas previstas \\
\hline $\begin{array}{c}\text { 1. Recordar } \\
\text { información }\end{array}$ & $\begin{array}{l}\text { Recordar hechos y conceptos; por ejemplo la localización } \\
\text { de topónimos en un mapa mudo o recordar la definición } \\
\text { de un concepto aislado }\end{array}$ \\
\hline $\begin{array}{c}\text { 2. Entender la } \\
\text { información }\end{array}$ & $\begin{array}{l}\text { Comprender y trasladar su conocimiento a nuevas ideas } \\
\text { realizando por ejemplo comparaciones y comprobaciones } \\
\text { de hechos geográficos }\end{array}$ \\
\hline $\begin{array}{c}\text { 3. Aplicar } \\
\text { conocimientos }\end{array}$ & $\begin{array}{l}\text { Realizar hipótesis, solucionar problemas usando sus } \\
\text { habilidades y conocimientos sobre la temática que está } \\
\text { tratando, utilización de datos con técnicas oportunas. }\end{array}$ \\
\hline $\begin{array}{c}\text { 4. Analizar } \\
\text { conocimientos }\end{array}$ & $\begin{array}{l}\text { Establecer relaciones, organizar, reconocer conceptos y } \\
\text { significados implícitos, identificar y clasificar cohesionada } \\
\text { y estructuradamente lo que pide el enunciado. Evaluar } \\
\text { sus hipótesis de partida. }\end{array}$ \\
\hline $\begin{array}{c}\text { 5. Juzgar. Evaluar } \\
\text { conocimiento }\end{array}$ & $\begin{array}{l}\text { Comparar, criticar, juzgar, realizar argumentaciones sobre } \\
\text { la organización territorial o sobre la manera de solucionar } \\
\text { un problema geográfico. }\end{array}$ \\
\hline
\end{tabular}

Fuente: Elaboración propia sobre Bloom en Anderson y Krathwolhl (2001)

Esta gradación de dificultad en la elaboración de los argumentos y razonamientos geográficos nos ha permitido clasificar el tipo de preguntas que se proponen en los exámenes de las PAU de las Comunidades Autónomas, completando así la descripción que hemos hecho anteriormente con un primer análisis tipológico. La selección de casos es el que aparece en el cuadro II. 


\section{Cuadro II. Clasificación de las preguntas de los exámenes de las PAU de Geografía}

\begin{tabular}{|c|c|}
\hline NIVELES DE BLOOM & PREGUNTAS \\
\hline 1 (Recordar Información) & $\begin{array}{c}1 \mathrm{CV}, 1 \mathrm{C}, 2 \mathrm{C}, 1 \mathrm{M}, 2 \mathrm{M}, 4 \mathrm{M}, 1 \mathrm{MU}, 2 \mathrm{MU}, 2 \mathrm{CL}, 3 \mathrm{CL}, 4 \mathrm{CL} \\
1 \mathrm{BAL}, 2 \mathrm{BAL}\end{array}$ \\
\hline 2 (Entender la información) & $3 \mathrm{M}, 3 \mathrm{BAL}$ \\
\hline 3 (Aplicación de los conocimientos) & $2 \mathrm{CV}, 4 \mathrm{C}, 1 \mathrm{CL}, 4 \mathrm{BAL}, 3 \mathrm{MU}$ \\
\hline 4 (Analizar los conocimientos) & $3 \mathrm{CV}, 3 \mathrm{C}$ \\
\hline 5 (Evaluación de las hipótesis) & $4 \mathrm{CV}$ \\
\hline $\begin{array}{l}\text { CV= Comunidad Valenciana, Junio } \\
\mathrm{C}=\text { Cataluña, Junio } 2012 \\
\text { M= Comunidad de Madrid, Junio } 2\end{array}$ & $\begin{array}{l}\text { BAL }=\text { Baleares Junio, } 2012 \\
\text { CL= Castilla León, Junio } 2012 \\
\text { MU = Murcia, Junio } 2012\end{array}$ \\
\hline
\end{tabular}

Fuente. Elaboración propia

Como se puede observar en el cuadro II la mayor parte de las preguntas que se proponen en los exámenes de las PAU de Geografía, según la muestra analizada, corresponden a niveles cognoscitivos bajos. Tan sólo en el caso de Cataluña y Comunidad Valenciana podemos apreciar algunas cuestiones que suponen un nivel más elevado y que representan el análisis y la valoración. Ello nos corrobora la hipótesis que venimos manejando: las PAU no suponen una valoración de los niveles de aprendizaje del alumnado (pues no se llegan a cuestionar), sino más bien una clasificación de éstos en relación con unas calificaciones numéricas basadas en un control cognoscitivo bajo.
Por su parte, la taxonomía SOLO ha sido utilizada para analizar el nivel cognoscitivo de los estudiantes en cada pregunta del examen. Hemos visto que en la prueba de Valencia las preguntas aumentan su complejidad progresivamente, por lo que queremos analizar como lo aplican los alumnos. Los niveles taxonómicos considerados son los siguientes (Cuadro III).

Una vez realizado el análisis examen por examen con las taxonomías citadas anteriormente, tenemos que demostrar la validez del estudio. Para ello, fue necesario eliminar la influencia del azar que se puede producir al realizar el análisis cualitativo, por lo que solicitamos el criterio de otro observador ${ }^{12}$. La téc-

\footnotetext{
12 Para ello hemos utilizado a otro investigador, Carlos Fuster García, que estaba realizando su TFM con el análisis de las PAU de Historia. Ello nos ha permitido verificar el acuerdo conseguido en la valoración de las respuestas del alumnado.
} 


\section{Cuadro III. Niveles taxonómicos de SOLO}

\begin{tabular}{|l|l|}
\hline Niveles jerárquicos & Tareas que suelen realizar los alumnos \\
\hline Pre-estructural & $\begin{array}{l}\text { Los alumnos recuerdan y reconocen información específica, sin } \\
\text { embargo no saben cómo desarrollarla por lo que suelen volcar infor- } \\
\text { mación sin organización, sentido y estructura. No comprenden lo } \\
\text { que han estudiado y se limitan a volcar información sin relación ni } \\
\text { sentido. }\end{array}$ \\
\hline Uni-estructural & $\begin{array}{l}\text { Los estudiantes son capaces de conectar ideas simples y obvias pero } \\
\text { continúa sin conocer el significado. Como característica observada en } \\
\text { este nivel es que los estudiantes ya conocen la información y recono- } \\
\text { cen conceptos, sin embargo no saben explicarlos bien, parafrasean, } \\
\text { confunden unos contenidos con otros }\end{array}$ \\
\hline Multi-estructural & $\begin{array}{l}\text { Pueden relacionar conceptos de diversos ámbitos geográficos pero les } \\
\text { falta la capacidad de síntesis y cohesión. La característica común de } \\
\text { los estudiantes en este nivel es que son capaces de resolver, aplicar } \\
\text { o calcular pero no de relacionar. Por ejemplo, son capaces de realizar } \\
\text { un gráfico pero fallan en su explicación. }\end{array}$ \\
\hline Relacional & $\begin{array}{l}\text { Son capaces de elaborar una explicación en la que relacionan con- } \\
\text { tenidos y los explican en relación con los temas principales. Como } \\
\text { característica de este nivel, los estudiantes son capaces de clasificar y } \\
\text { seleccionar el contenido que más le conviene, con una gran capacidad } \\
\text { de síntesis e interpretación de la temática tratada. }\end{array}$ \\
\hline Abstracto ampliado & $\begin{array}{l}\text { Es capaz de crear principios e ideas y generalizar. Demuestra que } \\
\text { sabe más de lo que le pide el enunciado por lo que una característica } \\
\text { de este nivel en los estudiantes es que tienen grandes conocimientos } \\
\text { sobre la materia por lo que también son capaces de establecer una } \\
\text { crítica, juzgar, diseñar, mejorar, etc. }\end{array}$ \\
\hline
\end{tabular}

Fuente: Elaboración Propia.

nica que hemos empleado para reducir la concordancia entre los observadores nos permite eliminar la subjetividad. Es una medida de concordancia para variables cualitativas conocida como el "coeficiente Kappa de Cohen”. Esta técnica calcula la diferencia entre la proporción de acuerdo observado y la proporción de acuerdo esperado al azar. Con esta técnica tratamos de medir el grado de acuerdo entre tres observadores que clasifiquen tanto las cuestiones del examen 
como las respuestas del alumno en una muestra común de 20 exámenes de geografía, comparando nuestros resultados con los del observador solicitado.

\section{Análisis de respuestas del alumnado e informes de correctores}

Tanto el ejercicio 1 como el 2 constan de 4 preguntas ordenadas ascendentemente según su grado de dificultad, sin embargo a todas se le otorga la misma puntuación (2,5 puntos), lo cual es obje- table, como hemos dicho y precisaremos en el momento de las conclusiones.

A partir de la taxonomía SOLO hemos analizado las respuestas de los alumnos con el objetivo de explicar los errores e insuficiencias detectados, para más tarde compararlos con los criterios de corrección exigidos y mejoras que sugieren los correctores y nosotros mismos. Respecto al ejercicio 1 podemos sintetizar estos elementos en el cuadro 4, que complementa la información aportada en el epígrafe 2.

\section{Cuadro IV. Errores y criterios de valoración del Ejercicio 1}

\begin{tabular}{|c|c|c|c|}
\hline ACTIV. & $\begin{array}{l}\text { PROBLEMAS } \\
\text { DETECTADOS }\end{array}$ & $\begin{array}{l}\text { CRITERIOS DE } \\
\text { CORRECCIÓN }\end{array}$ & $\begin{array}{l}\text { MEJORAS } \\
\text { SUGERIDAS }\end{array}$ \\
\hline 1 & $\begin{array}{c}\text { Mapas en blanco, incompletos } 0 \\
\text { con bastantes errores }\end{array}$ & $\begin{array}{l}\text { Localizar en mapa: relieve } \\
\text { peninsular (topónimos y } \\
\text { tipos de roquedo) }\end{array}$ & $\begin{array}{l}\text { Relacionar las dos variables } \\
\text { y realizar una explicación }\end{array}$ \\
\hline 2 & $\begin{array}{c}\text { No saben interpretar, } \\
\text { simplemente vuelcan los datos } \\
\text { de la tabla y confunden unos } \\
\text { climas con otros }\end{array}$ & $\begin{array}{l}\text { Identificar dos tipos de clima } \\
\text { a partir de los datos adjuntos } \\
\text { y explicar diferencias de } \\
\text { ambos climas. Razonar las } \\
\text { explicaciones. }\end{array}$ & $\begin{array}{l}\text { Añadir en el enunciado que } \\
\text { el alumno razone bien sus } \\
\text { explicaciones, relacionando } \\
\text { elementos }\end{array}$ \\
\hline 3 & $\begin{array}{c}\text { Confunden una vertiente con la } \\
\text { otra, faltan características para } \\
\text { poder tener toda la puntuación } \\
\text { de la actividad }\end{array}$ & $\begin{array}{l}\text { Realizar una diferenciación } \\
\text { (vertiente atlántica y } \\
\text { mediterránea) a partir de } \\
\text { los datos de las actividades } \\
\text { anteriores }\end{array}$ & $\begin{array}{c}\text { Insistir en la utilización de } \\
\text { datos que dan las anteriores } \\
\text { actividades para definir el } \\
\text { hilo conductor }\end{array}$ \\
\hline 4 & $\begin{array}{c}\text { No entienden la pregunta o } \\
\text { no la saben contestar porque } \\
\text { no responden lo que se les } \\
\text { pregunta. Tienen problemas } \\
\text { para organizar el discurso. } \\
\text { Dificultades para sistematizar } \\
\text { las conclusiones. }\end{array}$ & $\begin{array}{l}\text { Realizar una redacción, } \\
\text { recopilando toda la } \\
\text { información que nos dan las } \\
\text { actividades anteriores }\end{array}$ & $\begin{array}{l}\text { Mejorar la forma de } \\
\text { preguntar. Por ejemplo: } \\
\text { "Apoyándose en las anteriores } \\
\text { actividades, explique los } \\
\text { factores que influyen en el } \\
\text { caudal de los ríos españoles" }\end{array}$ \\
\hline
\end{tabular}

Fuente: Elaboración Propia 
Cuando aplicamos la taxonomía SOLO al análisis de las respuestas de los alumnos a las preguntas del Ejercicio 1 hemos comprobado que la mayoría de aquellas no superaban el nivel multiestructural, por lo cual una de las características esenciales del conocimiento geográfico, como es la capacidad de relacionar e integrar variables en una explicación sistémica no se alcanzaba. El cuadro V nos permite visualizar las dificultades que tienen los alumnos para llegar a los niveles superiores de razonamiento geográfico.

\section{Cuadro V. Niveles taxonómicos de SOLO en las actividades del Ejercicio 1}

\begin{tabular}{|lrrrr|}
\hline & \multicolumn{4}{c|}{ Actividades } \\
\hline Niveles SOLO & $\mathbf{1}^{\mathbf{a}}$ & $\mathbf{2}^{\mathbf{a}}$ & $\mathbf{3}^{\mathbf{a}}$ & $\mathbf{4}^{\mathbf{a}}$ \\
\hline Inferior al preestructural & 3 & 1 & 2 & 3 \\
\hline Preestructural & $\mathbf{1 2}$ & $\mathbf{9}$ & 6 & 4 \\
\hline Uniestructural & $\mathbf{1 6}$ & 5 & $\mathbf{1 0}$ & $\mathbf{8}$ \\
\hline Multiestructural & 5 & 11 & 11 & 11 \\
\hline Relacional & 1 & 7 & 6 & 8 \\
\hline Abstracto/ampliado & 0 & 4 & 2 & 3 \\
\hline
\end{tabular}

Fuente: Elaboración Propia

Esta tabla muestra la cantidad de alumnos que han llegado en cada actividad de la prueba, a cada nivel de la Taxonomía de SOLO. Hemos añadido el nivel inferior al pre-estructural para incluir las respuestas en blanco o los casos en los que toda la respuesta es errónea. A simple vista, podemos observar que la mayor cantidad de alumnos por nivel se encuentran entre los niveles Uniestructural y Multiestructural.

Analizando cada actividad, vemos que en la primera, la mayor parte de los alumnos se encuentran entre el Preestructural y el Uniestructural. Esto es debido a que los alumnos no pueden llegar a los niveles más elevados debido a que en la actividad no se les exige. En el nivel Preestructural la mayoría de estudiantes no completaban el mapa correctamente o sólo realizaban una de las dos partes de la pregunta. Sólo hay un alumno que voluntariamente realizó una pequeña relación entre las unidades de relieve y el tipo de roquedo.

En la segunda actividad, la mayor parte de estudiantes se encuentran en los niveles Multiestructural, Preestructural y relacional. En el caso del nivel Multiestructural, se debe a que detectan el clima correspondiente a los datos climáticos que se les ofrece, sin embargo no son capaces de explicar las características, relacionándolo y razonándolo con esos datos. Por otro lado, también existe abundancia en el nivel Preestructural y esto es debido a que muchos "vuelcan la información", es decir explican el clima que mejor habían aprendido sin sentido y sin relacionarlo con los datos de la tabla. También destacamos el nivel relacional puesto que lo alcanzan siete alumnos demostrando que son capaces de llegar a los niveles más elevados.

Finalmente, tanto en la tercera cuestión como en la cuarta pregunta la mayoría de estudiantes se centran en los nive- 
les Uniestructural y Multiestructural de la taxonomía SOLO. Es decir, con estos resultados vemos que los estudiantes están preparados para superar la prueba de geografía pero les falta un poco más de rigor para conseguir superar los niveles más elevados de la taxonomía.

En el Ejercicio 2 hemos procedido de forma semejante al primer Ejercicio, de tal manera que el cuadro VI nos refleja los errores, criterios de corrección señalados por la comisión y las mejoras que se sugieren, que serán precisadas en las conclusiones.

La primera pregunta está planteada con el nivel más bajo de la taxonomía de Bloom (Recordar y/o memorizar). La segunda pregunta, está planteada para que el alumno la responda con un mayor nivel cognitivo, concretamente para ser

\section{Cuadro VI. Errores y criterios de valoración del Ejercicio 2}

\begin{tabular}{|c|c|c|c|}
\hline ACTIV. & $\begin{array}{l}\text { PROBLEMAS } \\
\text { DETECTADOS }\end{array}$ & $\begin{array}{l}\text { CRITERIOS DE } \\
\text { CORRECCIÓN }\end{array}$ & $\begin{array}{l}\text { MEJORAS } \\
\text { SUGERIDAS }\end{array}$ \\
\hline 1 & $\begin{array}{c}\text { Gráficos sin título. } \\
\text { Gráficos mal estructurados, de } \\
\text { difícil lectura }\end{array}$ & $\begin{array}{l}\text { La construcción } \\
\text { ortodoxa de un gráfico } \\
\text { de barras. }\end{array}$ & $\begin{array}{l}\text { El alumno escoge la técnica de } \\
\text { representación geográfica. }\end{array}$ \\
\hline 2 & $\begin{array}{l}\text { Confunden factores sociales } \\
\text { como factores físicos }\end{array}$ & $\begin{array}{l}\text { Características que } \\
\text { explican el predominio } \\
\text { de la carretera en los } \\
\text { medios de transporte. }\end{array}$ & $\begin{array}{l}\text { Puntuar según estos criterios: } \\
\text { 1) Tipos de transporte. } \\
\text { 2) Características del transporte } \\
\text { por carretera. } \\
\text { 3) Densidad de carreteras. } \\
\text { 4) Red Radial. } \\
\text { 5) Fondos europeos. }\end{array}$ \\
\hline 3 & $\begin{array}{c}\text { Confundir el ferrocarril como } \\
\text { medio de trasporte más } \\
\text { utilizado. }\end{array}$ & $\begin{array}{l}\text { Comprender las } \\
\text { características y la } \\
\text { distribución de las } \\
\text { principales redes de } \\
\text { ferrocarril. }\end{array}$ & $\begin{array}{l}\text { Comparar el ferrocarril con el } \\
\text { transporte aéreo y marítimo. } \\
\text { Comentar ventajas-desventajas, y } \\
\text { cómo interactúan con el transporte } \\
\text { Intermodal. }\end{array}$ \\
\hline 4 & $\begin{array}{l}\text { Se generaliza excesivamente } \\
\text { en lugar de reflexionar sobre lo } \\
\text { mejor que puede aportar cada } \\
\text { medio de trasporte, así como } \\
\text { ventajas y desventajas comunes } \\
\text { para elaborar un texto mucho } \\
\text { más cohesionado. }\end{array}$ & $\begin{array}{c}\text { 1) Crecimiento } \\
\text { espectacular durante } \\
\text { el s.XX } \\
\text { 2) Nuevas Tendencias } \\
\text { 3) Principales } \\
\text { Corredores. }\end{array}$ & $\begin{array}{c}\text { Coletilla final: "Si es capaz, } \\
\text { proponga posibles soluciones a } \\
\text { dichos desequilibrios territoriales y } \\
\text { añada algún ejemplo que conozca". }\end{array}$ \\
\hline
\end{tabular}

Fuente: Elaboración Propia 
contestada al menos con el nivel 3 de la taxonomía (realizar un texto cohesionado, relacionando sus ideas con los conceptos en cuestión). A su vez la tercera pregunta está planteada para ser respondida como mínimo con el nivel 4 de la taxonomía (el estudiante es capaz de apreciar el significado de las partes en relación con el conjunto),

La pregunta número cuatro se trata de una pregunta abierta y no como las anteriores que se encuentran cerradas en base a cuestiones más concretas. Por ello la pregunta tiene por objeto ser respondida con el mayor nivel cognitivo que pueda aportar el alumno, lo cual según la taxonomía de Bloom le corresponde un nivel de evaluación de las hipótesis (nivel 5), estableciendo el objetivo de valorar el problema según sus conocimientos y criterios, que muestre su opinión si estima oportuno e incluso, que vaya más allá en su explicación relacionando el problema con otras temáticas que pudiera observar que comparten características o finalidades comunes.

Sin embargo, como podemos apreciar en el cuadro VII son escasas las personas que alcanzan los niveles 4 y 5 de la taxonomía SOLO, lo que nos sugiere que habrá que mejorar la redacción de las preguntas y la manera de trabajar en las aulas, como se indicará.

Como podemos observar, en la pregunta uno la mayoría han llegado al nivel 3 , por lo que la pregunta ha cumplido su objetivo ya que estaba destinada a ser contestada con ese nivel, sin embargo, hay que observar la cantidad de alumnos que se han quedado en el nivel dos.

\section{Cuadro VII. Niveles taxonómicos de SOLO en las actividades del Ejercicio 2}

\begin{tabular}{|lrrrrr|}
\hline & \multicolumn{4}{c|}{ Actividades } \\
\hline Niveles SOLO & $\mathbf{1}^{\mathbf{a}}$ & $\mathbf{2}^{\mathbf{a}}$ & $\mathbf{3}^{\mathbf{a}}$ & $\mathbf{4}^{\mathbf{a}}$ \\
\hline Inferior al preestructural & 5 & 13 & 8 & 12 \\
\hline Preestructural (nv.1) & 22 & $\mathbf{1 6}$ & $\mathbf{1 2}$ & $\mathbf{1 0}$ \\
\hline Uniestructural (nv.2) & $\mathbf{1 6}$ & 5 & 10 & 8 \\
\hline Multiestructural (nv.3) & 29 & 14 & 18 & 19 \\
\hline Relacional (nv.4) & 0 & 11 & 13 & 11 \\
\hline Abstracto/ampliado (nv.5) & 0 & 3 & 3 & 2 \\
\hline
\end{tabular}

Fuente: Elaboración Propia

Esto se debe a que se cometen muchos fallos importantes como olvidar el título del gráfico o estructurarlo mal (aspectos que los docentes debemos reflexionar).

En la segunda pregunta resulta preocupante observar un retroceso cognitivo en su contestación porque estaba destinada a ser contestada con un nivel mínimo de tres. Lo cual significa que hay que reflexionar, como afirmábamos antes, de que quizá sería oportuno mejorar este nivel facilitándoles a los alumnos algunas pistas sobre los conceptos que debería relacionar. Por último, comentamos las dos últimas preguntas en base a una observación importante. Véase como a pesar de que la mayoría de respuestas son de nivel 3, existe un número importante de respuestas en torno al nivel relacional (nivel mínimo con el que estaban objetivadas ambas preguntas). Esto podría explicar que quizá algunos de estos alumnos podrían haber llegado 
al nivel 5 pero no lo han hecho porque probablemente no se han visto motivados por el planteamiento de la pregunta. Tal vez, reformulando la pregunta, esta mejoraría sus niveles y cumpliría mejor su objetivo final, que es diagnosticar hasta cuanto de significativo ha resultado ser el aprendizaje de los alumnos.

Por su parte, los correctores de las PAU no hacen generalmente un informe de los problemas detectados, pero cuando ello sucede podemos comprobar que se aproximan al diagnóstico aquí referido, así como a otros comentarios que se han recogido en el documento editado por el Departamento de Geografía de la Universitat de Valencia (Obiol, Salom y Souto, 2008).

Así subrayan la existencia de problemas formales, tales como errores en la sintaxis, léxico y ortografía en algunos alumnos. En este sentido, también se indica que adolecen del uso de conceptos básicos para explicar los problemas. Por ejemplo, ninguno ha utilizado el concepto de disimetría hídrica entre las cuencas atlánticas y mediterráneas. Menos de un $10 \%$ hace referencia a las cuencas hidrográficas, que incluyen los ríos con sus afluentes, lo que era importante para explicar el caudal de los ríos ${ }^{13}$. Igualmente menos del $10 \%$ ha utilizado los conceptos de ejes de transporte (como los que se señalaban en las correcciones) e intermodalidad.

La Intensidad Media Diaria ha sido definida correctamente por 28 personas y 34 lo han hecho mal. En el caso de la densidad de carreteras los resultados son peores: 11 bien y 51 mal. Seguramente era una cuestión muy concreta y que no se ha trabajado en clase, pues se observa que los alumnos que lo han hecho bien lo hacían por deducción, lo cual muestra una gran madurez del alumnado, pues sabían relacionar asentamientos y transporte por carretera. En este sentido se comprueba que el aprendizaje de geografía dispone de un refuerzo muy importante en la construcción conceptual como es la aportación de datos procedentes de la vida cotidiana.

En la confección de la gráfica todavía un número importante ( 27 de 62 ) no han colocado título en la figura de barras que han elaborado. En la localización del mapa de la pregunta 1 registramos 31 mapas con errores (de 51) y sólo 10 que se hayan equivocado en la leyenda litológica.

Sin embargo, la falta de organización de los conceptos y la ausencia de datos empíricos para razonar ha sido muy importante en el momento de redactar las preguntas número 4 , en la cual se esperaba una conclusión sobre los factores del caudal fluvial o sobre los desequilibrios, nuevas tendencias y ejes y redes de transporte.

\section{Conclusiones}

Después de haber realizado este doble análisis, tanto de las respuestas

${ }^{13}$ Seguimos el informe elaborado por el corrector del Tribunal 12 de la PAU de Geografía de la Universidad de Valencia en la convocatoria de junio de 2012. 
de los alumnos como de las actividades propuestas y conociendo los distintos modelos de PAU en España, vemos que el caso de la Comunidad Valenciana es distinto a otras comunidades ya que las actividades siguen una lógica ascendente de niveles cognitivos.

\section{Sobre las posibilidades de aprendizaje} del alumnado.

El estudio de las respuestas que ha realizado el alumnado en las PAU de Geografía de la Comunidad Valenciana nos ha permitido comprender las limitaciones de su aprendizaje. Así en el caso de la segunda pregunta del Ejercicio 1 no sólo necesita tener conocimientos previos, sino que además tiene que comprender los datos y poder identificar el tipo de clima al que pertenecen y a partir de ahí realizar la descripción. Se trata de una actividad muy completa y en el que el alumno puede demostrar muy bien sus conocimientos sobre esta materia, pero que requiere de un dominio conceptual de los tres elementos que aparecen en el ejercicio.

Así hemos comprobado que existe un numeroso grupo de alumnos en el nivel preestructural y esto es debido a que muchos "vuelcan la información", es decir explican el clima que mejor habían aprendido sin sentido y sin relacionarlo con los datos de la tabla. También destacamos el nivel relacional puesto que lo alcanzan siete alumnos demostrando que son capaces de llegar a los niveles más elevados.
Por otra parte, registramos aspectos positivos, de incorporación de nueva información, más allá de la que se solicitaba en los criterios de corrección:

a) La localización de los mapas suele ser correcta, incluyendo más información que la solicitada (p.e. Sierra Tramuntana en Balears)

b) Información sobre trasvases, consumo de agua y contaminación del caudal en la pregunta de los ríos, pero no saben utilizar los mapas conceptuales para ordenar sus ideas.

\section{Cómo mejorar la formulación de} preguntas.

Hemos observado que en algunas ocasiones se podría mejorar las respuestas del alumnado si las preguntas facilitaran la relación de elementos. Un ejemplo de lo que queremos decir sería la pregunta 3 del Ejercicio 2, pues se podría pedir al alumno comparar las características que ha explicado del ferrocarril contrastadas con las de otro medio de transporte que, considerando que ya se le ha preguntado por el medio terrestre, se le exigiera comparar con el transporte marítimo o el aéreo de manera que pudiera o bien contrastar ventajas y desventajas, o bien contrastar costes en base al tipo de mercancía y la distancia a recorrer (como variables importantes). Para comprender mejor esta propuesta, reformularíamos así la pregunta: " $E n$ base a sus conocimientos sobre las características y distribución de las redes de transporte nacional de cada modo de 
transporte, Si usted tuviera que enviar un cargamento con una tara o peso entre 1-4 toneladas desde su ciudad natal de Zaragoza hasta la ciudad costera de Venecia, ¿Qué tipos de transportes cree usted que tendría disponibles y resultarían más rentables para cada una de las distancias a recorrer? y relacione su explicación con la importancia del transporte intermodal". De este modo aparece un marcado concepto clave sobre el que los alumnos podrían apoyar su respuesta. Además se trataría de una pregunta distinta que a los alumnos les serviría para que no se repitieran sobre conceptos $\mathrm{u}$ explicaciones anteriores, aportando además, un cambio de escala en la concepción del alumno sobre los medios de transporte, que probablemente le servirá para alimentar su reflexión final en la última pregunta. Qué podemos proponer como profesores-correctores. Para el futuro parece preciso incidir más en las relaciones conceptuales para alcanzar una explicación sistémica, propia de la geografía. En este sentido el examen puede ayudar siempre que se mantenga la pregunta 4 como síntesis de las cuestiones anteriores y facilite la interpretación de una situación social con la ayuda de los conceptos y los datos expuestos en los documentos del examen. En este sentido es muy importante mantener la lógica del examen:

a) Análisis de una información de un documento o construcción de un gráfico o mapa.

b) Explicación de los documentos con los conceptos que están insertos en los mismos.

c) Relación de conceptos y datos para explicar una situación geográfica.

Por ejemplo podían utilizar algo semejante a lo siguiente:

\section{Cuadro VIII. Ejemplo de lógica de examen para el Ejercicio 1 u opción A}

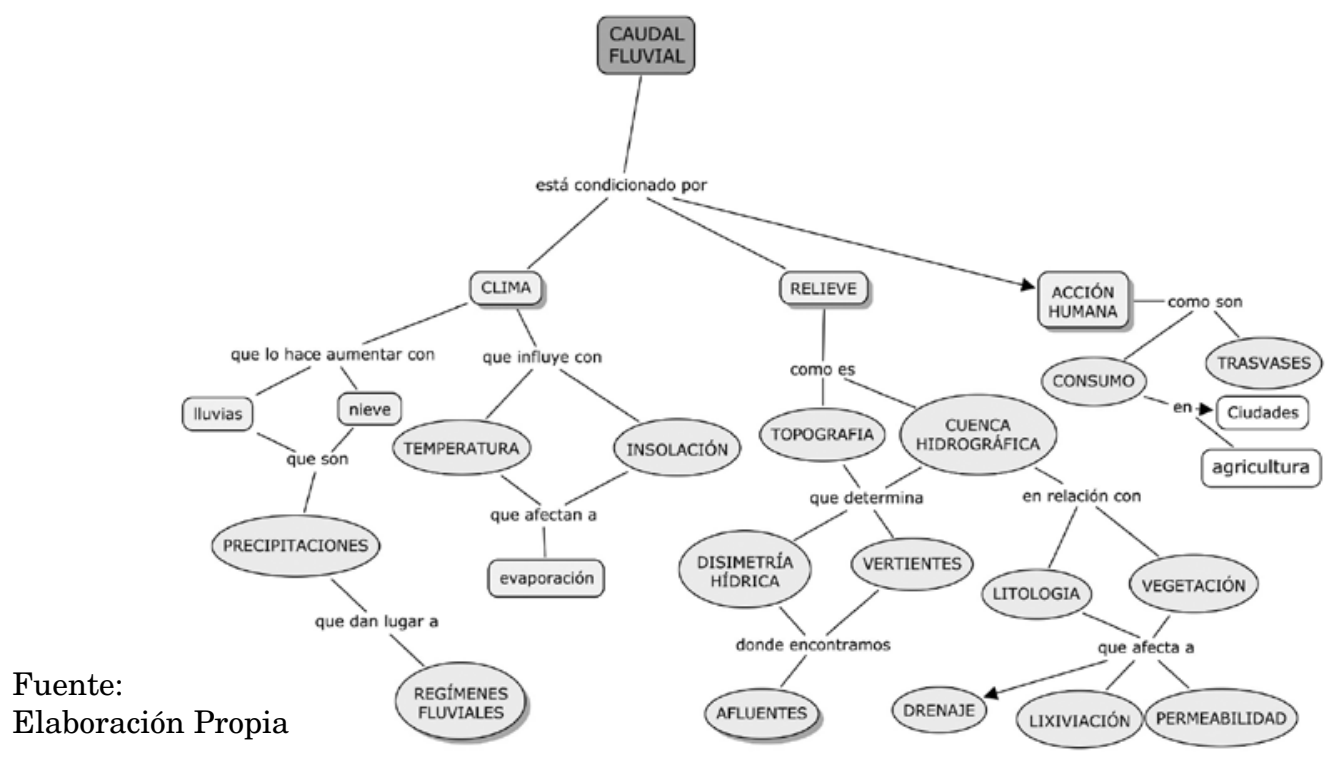




\section{Cuadro IX. Ejemplo de lógica de examen para el Ejercicio 2 u opción B}

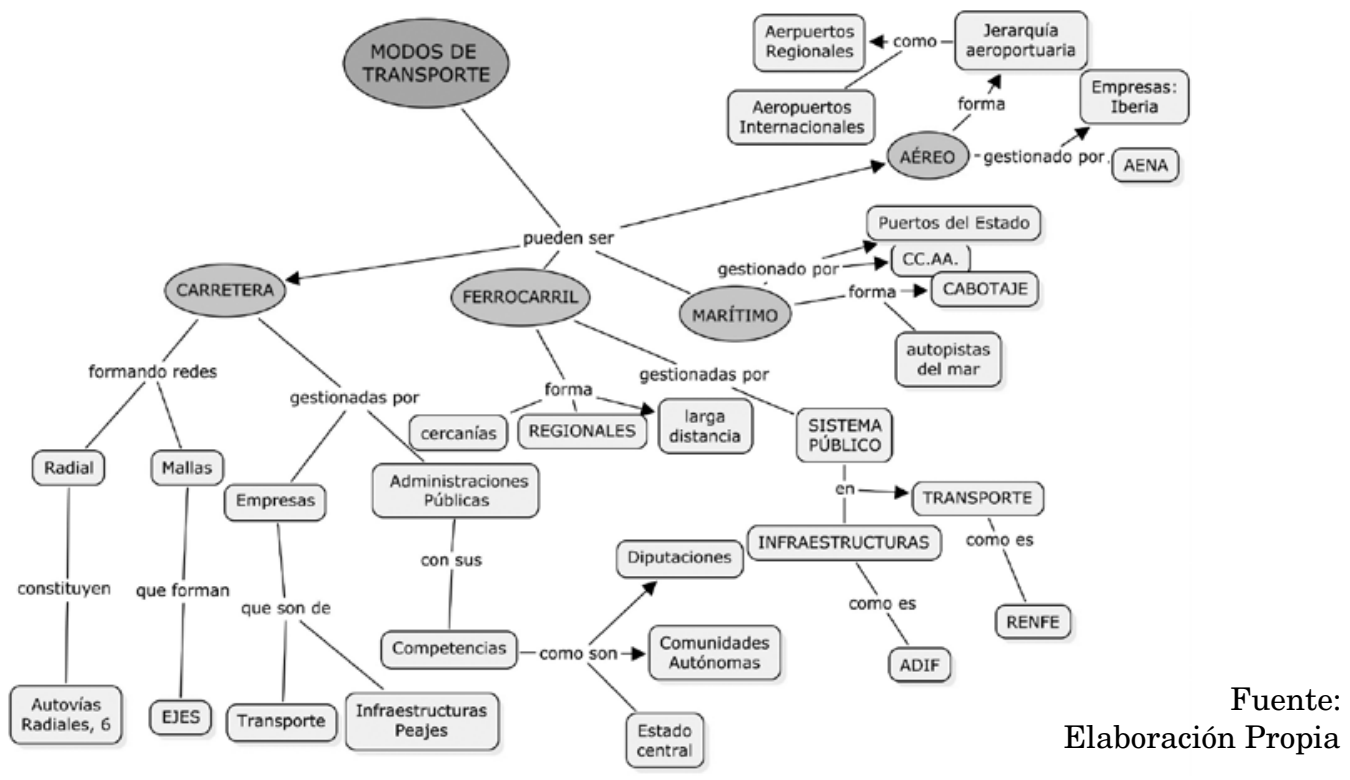

Dichas conclusiones nos animan a proseguir con un estudio de los exámenes y otros instrumentos de evaluación no sólo en el Bachillerato, sino en toda la educación básica, en línea con lo que ya vienen trabajando otros colegas (Miralles et al., 2012).

\section{Referencias bibliográficas}

ANDERSON, W., and KRATHWOHL, David R., (eds. 2001). A Taxonomy for Learning, Teaching, and Assessing: A Revision of Bloom's Taxonomy of Educational Objectives. New York: Longman.

CLAUDINO, S.; SOUTO, X.M. (2001). Obstáculos en la innovación de la didáctica de la geografía. En Marrón Gaite, M. ${ }^{a}$ J. (ed.). La formación geo- gráfica de los ciudadanos en el cambio de milenio.(p. 191-203). Madrid:AGEAPGP-Universidad Complutense.

CLIMENT LÓPEZ, E. (2001). Las Pruebas de Acceso a la Universidad y la enseñanza de la geografía en el bachillerato español, en MARRÓN GAITE, María Jesús (editora). La formación geográfica de los ciudadanos en el cambio del milenio, (p.1976-1985). Madrid: Asociación de Geógrafos Españoles, Associação de Profesores de Geografía de Portugal y Universidad Complutense de Madrid.

HOUTSONEN, Lea (1988). The reform of geography teaching in the Finish upper secundary school and its reflection. The Matriculation Examinations of 1976-1985, Fennia, 166, 2, 413-570. 
IBARRA BENLLOCH, P. y LACOSTA ARAGÜÉS, A. (2010). Renovación de objetivos y planteamientos metodológicos innovadores en las pruebas de acceso a las enseñanzas universitarias (PAEU) de Geografía de la Universidad de Zaragoza. En MARRÓN GAITE, M ${ }^{a}$ J. y DE LÁZARO Y TORRES, $M^{a}$ L. (Eds.): Geografía, Educación y Formación del Profesorado en el Marco del espacio Europeo de Educación Superior (2 vols.). (p. 611-619); (385-403). Murcia: Grupo de Didáctica de la Geografía de la Asociación de Geógrafos Españoles y Universidad Complutense de Madrid.

MARTÍN MARTÍN, J.; VÁZQUEZ SÁNCHEZ, $M^{a}$ L. (2011). La geografía en las pruebas de selectividad (PAEG), en DELGADO PEÑA, J.I.; DE LÁZARO TORRES, Ma la; MARRÓN GAITE, M ${ }^{\mathrm{a}}$ J. Aportaciones de la Geografía en el aprendizaje a lo largo de la vida. Actas del Congreso Ibérico de Geografía. (p. 670-677). Málaga: Universidad y AGE.

MARTÍNEZ MEDIA, R. y GIL MESEGUER, E. (2011). Las Pruebas de selectividad de Geografía en el distrito único de Murcia, en DELGADO PEÑA, J.I.; DE LÁZARO TORRES, $\mathrm{M}^{\mathrm{a}}$ l.; MARRÓN GAITE, M ${ }^{\mathrm{a}} \mathrm{J}$. Aportaciones de la Geografía en el aprendizaje a lo largo de la vida. Actas del Congreso Ibérico de Geografía. (p. 660669) Málaga: Universidad y AGE.

MELO, J.E. (2013). "Seu futuro passa por aquí”: ENEM como prática ava- liativa, In TONINI, I.; KAERCHER, N.; LÓPES, F. Ensino da Geografia e da História. Saberes e fazeres na conptemporaneidade, (p. 195-214). Porto Alegre: UFRGS, Evangraf.

MESEJO GONZÁLEZ, C. (2007). Las pruebas de acceso a la Universidad vistas desde el Noroeste, en MARRÓN, M J J.; SALOM, J.; SOUTO, X.M. Las competencias geográficas para la educación ciudadana, (p. 449-456). Valencia: Grupo Didáctica de la AGE y Universitat de València.

MIRALLES MARTÍNEZ, P., GÓMEZ CARRASCO, C.J. y MONTEAGUDO FERNÁNDEZ, J. (2012). La evaluación de la competencias social y ciudadana en ciencias sociales al finalizar las etapas de Primara y Secundaria. Investigación en la escuela, 78, 19-30.

NAVARRO LUNA, J.; GIL SALVADOR, F. (2001). La prueba de acceso en la materia de Geografía en la Universidad de Sevilla (2001-2011), en DELGADO PEÑA, J.I.; DE LÁZARO TORRES, Mำ; MARRÓN GAITE, $\mathrm{M}^{\mathrm{a}} \mathrm{J}$. Aportaciones de la Geografía en el aprendizaje a lo largo de la vida. Actas del Congreso Ibérico de Geografía. (p.678-688). Málaga: Universidad y AGE.

OBIOL, E. SALOM, J. y SOUTO, X.M (2008). Geografía y Pruebas de Acceso a la Universidad. Departamento de Geografía de la Universitat de Valencia,1 CD.

QUIÑONERO FERNÁNDEZ, Francisco (2012). De la tabla estadística al mapa. Un problema para el alum- 
nado, Iber, Didáctica de las ciencias sociales, Geografía e Historia, n. 70, p. 105-115.

SOUTO GONZÁLEZ, Xosé M (2011). Las P.A.U. de Geografía: ¿un obstáculo o una oportunidad? En MIRALLES MARTÍNEZ, P; MOLINA PUCHE, S.; SANTISTEBAN FERNÁNDEZ, A. (eds.) La evaluación en el proceso de enseñanza y aprendizaje de las ciencias sociales, v. II. Murcia: Asociación de Profesores Universitarios de Didáctica de las Ciencias Sociales, p, 271-284.

SOUTO, Xosé, CLAUDINO, Sérgio (2009) "Exames de Geografia, programas e inovação didáctica" in $A$ Inteligência Geográfica na Educação do Século XXI. Lisboa, APROFGEO/ IGOT-UL/GDG-AGE, p. 21-30.

SOUTO, Xosé M. FUSTER, Carlos y SÁIZ, Jorge (2014). Un camino de ida y vuelta: reválidas y selectividad en las rutinas escolares de la enseñanza de Geografía e Historia. En PAGÉS, Joan y SANTISTEBAN, Antoni (eds) Una mirada al pasado $y$ un proyecto de futuro. Investigación e innovación en didáctica de las ciencias sociales, Vol. 2, Barcelona: AUPDCS y Universitat Autónoma Barcelona, páginas 157-165. 
